\title{
Importancia de la antibioticoterapia ante colecciones intraabdominales complicadas en pacientes con lesión renal aguda: reporte de casos
}

\author{
Importance of antibiotic therapy in complicated intra-abdominal \\ abscess in patients with acute kidney injury: case reports \\ Yeiscimin Sánchez-Escobedo, ${ }^{*}$ Mónica Isabel León-Morales, ${ }^{\ddagger}$ Roberto Ramírez-Vega ${ }^{\S}$
}

Palabras clave: Sepsis, infección intraabdominal, antibioticoterapia, enfermedad renal, lesión renal aguda.

Keywords: Sepsis, intraabdominal infection, antibiotic therapy, renal disease, acute kidney injury.

* Médico pasante del Servicio Social en Investigación. Facultad de Medicina. Benemérita Universidad Autónoma de Puebla.

‡ Médico Cirujano y Partero. Benemérita Universidad Autónoma de Puebla.

$\S$ Residente de Cirugía General. Hospital Universitario de Puebla.

Recibido: 08/08/2019 Aceptado: 15/02/2021

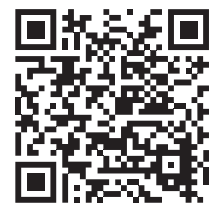

\section{RESUMEN}

Introducción: Las infecciones intraabdominales (IIA), cuando se asocian a lesión renal aguda, requieren modificaciones en la terapia antimicrobiana a emplear, tanto como iniciar de manera temprana y apropiada el antibiótico que cubra el espectro de gérmenes implicados. La importancia del control de la fuente repercute en el descenso de la tasa de mortalidad. Descripción de casos clínicos: Cinco pacientes con IIA complicada, cuyo valor medio de edad fue 55.6 años; $60 \%$ de esta población fueron hombres y $66 \%$ presentó comorbilidades como diabetes mellitus tipo dos, además de hipertensión arterial sistémica. En la mayoría de dichos sujetos, la presentación clínica fue dolor localizado y fiebre $(75 \%)$ y las escalas de gravedad de disfunción orgánica (un promedio de SOFA de 15.8 puntos y AKIN $80 \%$ con LRA tipo III). El antibiótico profiláctico prehospitalario más empleado fue la ceftriaxona y, de acuerdo al antibiograma, se decidió cambiar por alguno del grupo de carbapenémicos. En días, la duración media de esa terapia antimicrobiana fue de 23.4, sin embargo, la media del lapso de estancia intrahospitalaria fue de 18.2, lo cual sugiere un periodo de tiempo prolongado. Conclusión: La sepsis tiene incidencia y mortalidad elevadas. Por ello, la antibioticoterapia debe ser elegida de acuerdo a la gravedad y estado clínico, de laboratorio y de la disponibilidad de los fármacos, permitiendo que la decisión médica sea basada en evidencia.

\section{ABSTRACT}

Introduction: When intra-abdominal infections are associated with acute kidney injury, certain modifications are needed in relation to the antimicrobial therapy to be used, since the antibiotic that covers the spectrum of germs involved must also be indicated appropriately and early. The importance of source control has an impact on the decrease in the mortality rate. Description of clinical cases: Five patients having complicated intra-abdominal infection, whose mean age was 55.6 years, $60 \%$ of this population were men and $66 \%$ presented comorbidities as type two diabetes and systemic arterial hypertension. The clinical presentation most frequent in such subjects was localized pain and fever (75\%) and the severity scales of sepsis (average SOFA 15.8 points and AKIN $80 \%$ with LRA type III). The most frequently used prophylactic antibiotic in the pre-hospitalization stage was ceftriaxone and, once the antibiogram was performed, it was decided to switch to carbapenems. In days, the mean duration of antimicrobial therapy was 23.4; however, the average hospital stay was 18.2, which suggests a prolonged period of time. Conclusion: Sepsis has both high incidence and mortality; therefore, antibiotic treatment should be chosen according to the severity and clinical status, laboratory results and availability of drugs, allowing the medical decision to be based on evidence.

\section{Abreviaturas:}

IIA = Infección intraabdominal.

LRA = Lesión renal aguda.

$\mathrm{BLEE}=\beta$-lactamasa de espectro extendido.

AKIN = Acute Kidney Injury Network.

SOFA $=$ Sequential Organ Failure Assessments. qSOFA $=$ Quick SOFA.

\section{INTRODUCCIÓN}

$\mathrm{U}$ na infección intraabdominal (IIA) se considera complicada cuando se presenta de manera difusa o localizada dentro de la cavidad abdominal y tiene repercusión sistémica. ${ }^{1,2}$

Citar como: Sánchez-Escobedo Y, León-Morales MI, Ramírez-Vega R. Importancia de la antibioticoterapia ante colecciones intraabdominales complicadas en pacientes con lesión renal aguda: reporte de casos. Cir Gen. 2020; 42 (4): 300-305. https://dx.doi.org/10.35366/101399 
Se produce a consecuencia de la perforación o inflamación de la pared intestinal, y por lo tanto son contaminaciones propiciadas por muchos microorganismos, incluso llegan a ser mixtas (fúngicas o parasitarias), sin embargo, muestran un predominio causal de bacterias anaerobias. ${ }^{3,4}$

La presencia de sepsis predispone el desarrollo de lesión renal aguda (LRA), elevando aún más la tasa de mortalidad. ${ }^{5}$ Por ello, la importancia de elegir la terapia antibiótica adecuada en pacientes con LRA radica en que los antimicrobianos deben ajustarse a dosis capaces de alcanzar niveles séricos adecuados, evitando la sobredosis y la toxicidad de estos medicamentos, al igual que el riesgo de resistencia. ${ }^{4,5}$ Se han realizado diversos ensayos clínicos, los cuales concluyen que, de acuerdo a la tasa de filtración glomerular, se deben ajustar las dosis a fin de que sean efectivas, para que las drogas alcancen la concentración mínima inhibitoria y así logren su efecto bacteriostático o bactericida, según sea el caso, sin causar daño a los riñones. ${ }^{6,7}$

En la IIA complicada existen estrategias, como el control de la fuente mediante trata- miento quirúrgico y la elección de antimicrobianos, que se acompañan de índices de fracaso bajos y un descenso en la tasa de mortalidad. En México, un estudio multicéntrico acerca de sepsis reportó una mortandad de $30.4 \%$, siendo la etiología abdominal la más frecuente con $47 \%$, por ende, es de vital importancia contextualizar el caso de un paciente séptico con LRA, debido a que la evaluación de gravedad y la adopción de medidas terapéuticas urgentes conducen a una disminución del número de defunciones, de tal modo que cuando se controla con eficacia el origen y se utilizan antibióticos apropiados la tasa de respuesta favorable se ha registrado en 70 a $90 \% .^{8,9}$

\section{PRESENTACIÓN DE CASOS CLÍNICOS}

En el grupo bajo investigación se incluyeron cinco enfermos, quienes presentaron choque séptico de origen abdominal evidenciado mediante algún estudio de radiología asociado a LRA en cualquier estadio según la clasificación AKIN (Tabla 1). La edad media de presentación fue de 55.6 años, siendo relativamente más frecuente en hombres con $60 \%$. Las principales

Tabla 1: Características clínicas de la muestra estudiada. $\mathrm{N}=5$.

\begin{tabular}{|c|c|c|c|c|c|c|c|}
\hline Paciente & $\begin{array}{l}\text { Edad } \\
\text { (años) }\end{array}$ & Sexo & Comorbilidades & $\begin{array}{l}\text { Manifestación } \\
\text { clínica inicial }\end{array}$ & $\begin{array}{l}\text { Origen de } \\
\text { infección }\end{array}$ & $\begin{array}{l}\text { Diagnóstico } \\
\text { Inicial }\end{array}$ & $\begin{array}{l}\text { Diagnóstico } \\
\text { Final }\end{array}$ \\
\hline 1 & 64 & Femenino & DT2/HAS & $\begin{array}{l}\text { Dolor en hipocondrio } \\
\text { derecho, fiebre, } \\
\text { náusea, vómito }\end{array}$ & Vesícula biliar & $\begin{array}{l}\text { Colangitis severa } \\
\text { + colecistitis aguda } \\
\text { (Tokio grado III) }\end{array}$ & Piocolecisto \\
\hline 2 & 52 & Masculino & Alcoholismo & $\begin{array}{c}\text { Dolor en mesogastrio, } \\
\text { náusea y fiebre }\end{array}$ & Páncreas & $\begin{array}{l}\text { Pancreatitis aguda } \\
\text { severa } \\
\text { (Atlanta 2012) }\end{array}$ & $\begin{array}{c}\text { Pseudoquiste } \\
\text { pancreático } \\
\text { (tipo V) }\end{array}$ \\
\hline 3 & 54 & Masculino & DT2/alcoholismo & $\begin{array}{l}\text { Fiebre, ictericia, } \\
\text { dolor hipocondrio } \\
\text { derecho }\end{array}$ & Hígado & $\begin{array}{l}\text { Insuficiencia } \\
\text { hepática crónica } \\
\text { (Child-Pugh B) }\end{array}$ & $\begin{array}{l}\text { Abscesos } \\
\text { piógenos } \\
\text { hepáticos }\end{array}$ \\
\hline 4 & 67 & Femenino & DT2/HAS/IC & $\begin{array}{l}\text { Vómito, dolor en } \\
\text { fosa iliaca derecha }\end{array}$ & Riñón & $\begin{array}{c}\text { Hidronefrosis vs } \\
\text { pielonefritis + IVU } \\
\text { complicada }\end{array}$ & $\begin{array}{l}\text { Quiste renal } \\
\text { (Bosniak I) }\end{array}$ \\
\hline 5 & 41 & Masculino & HAS & $\begin{array}{l}\text { Fiebre, ictericia, } \\
\text { vómito }\end{array}$ & Vesícula biliar & $\begin{array}{c}\text { Colangitis severa + } \\
\text { coledocolitiasis }\end{array}$ & $\begin{array}{l}\text { Perforación } \\
\text { vesicular + } \\
\text { peritonitis }\end{array}$ \\
\hline
\end{tabular}

DT2 = diabetes tipo 2, HAS = hipertensión arterial sistémica, IC = insuficiencia cardiaca, IVU = infección de vías urinarias. 
Tabla 2: Evaluación hemodinámica y caracterización de lesión renal aguda. $\mathbf{N}=5$.

\begin{tabular}{|c|c|c|c|c|c|}
\hline \multirow[b]{2}{*}{ Paciente } & \multicolumn{3}{|c|}{ Disfunción orgánica } & \multicolumn{2}{|c|}{ Lesión renal aguda } \\
\hline & TAM inicial (mmHg) & SOFA-Score & qSOFA-Score & CSi (mg/dl) & CSf (mg/dl) \\
\hline 1 & 63.33 & 14.0 & 3.0 & 8.4 & 1.30 \\
\hline 2 & 60.00 & 12.0 & 2.0 & 3.1 & 1.00 \\
\hline 3 & 56.67 & 16.0 & 2.0 & 1.9 & 1.30 \\
\hline 4 & 50.00 & 20.0 & 3.0 & 12.9 & 3.20 \\
\hline 5 & 51.67 & 17.0 & 3.0 & 3.7 & 1.60 \\
\hline Promedio & 56.33 & 15.8 puntos & 2.6 puntos & 5.0 & 1.68 \\
\hline
\end{tabular}

TAM = tensión arterial media, SOFA = Sepsis related Organ Failure Assessment, qSOFA = Quick SOFA, CSi = creatinina sérica inicial, $\mathrm{CSf}=$ creatinina sérica final.

comorbilidades asociadas a una IIA complicada fueron: hipertensión arterial sistémica y diabetes tipo dos (ambas en 33\%), seguido de alcoholismo $(22.5 \%)$ y, por último, insuficiencia cardiaca (11.5\%). La presentación clínica más habitual fue la asociación de dolor localizado y fiebre. En el abordaje diagnóstico para determinar el estado hemodinámico e identificar la disfunción orgánica se utilizó la escala SOFA/ qSOFA con promedios de 15.8 y 2.6 puntos respectivamente; la tensión arterial media fue $56.33 \mathrm{mmHg}$ en todos los días de estancia hospitalaria. Los parámetros de laboratorio se elevaron en la totalidad de los pacientes y la mediana reportada fue: leucocitos $(26,040 \mathrm{cel} /$ $\left.\mathrm{mm}^{3}\right)$, procalcitonina $(87.6 \mathrm{pg} / \mathrm{ml})$, velocidad de sedimentación globular $(31.56 \mu \mathrm{g} / \mathrm{ml})$.

Se identificó y clasificó el grado de daño renal de acuerdo a los valores de creatinina en los primeros laboratorios, los de seguimiento y a su egreso, el resultado fue que $80 \%$ de los individuos se clasificaron en estadio III, según AKIN (Tabla 2). El estudio de imagen para iniciar el abordaje es el ultrasonido abdominal y a fin de valorar la evolución del paciente se solicitó TAC abdominopélvica (Figura 1).

El tratamiento médico se focalizó en dos puntos: el apoyo mediante fármacos vasopresores y la terapia antibiótica. El 60\% requirió aminas y respecto al empleo de medicamentos antimicrobianos el principal antibiótico empírico empleado fue la ceftriaxona (35.7\%) a
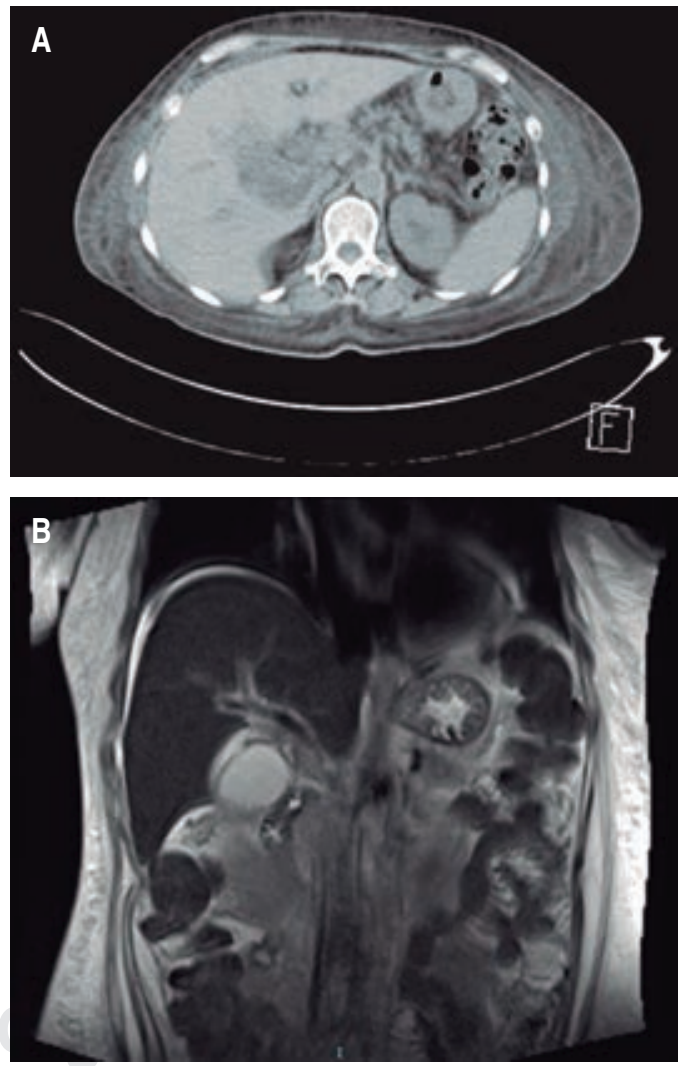

Figura 1: Estudios de imagen del paciente 1. A) Tomografía axial computarizada abdominal con evidencia de colecistitis aguda y líquido perivesicular sin poder descartar irrupción parcial de la pared, volumen de $184 \mathrm{~cm} 3$. B) Colangiorresonancia que evidencia colecistitis crónica agudizada, lodo biliar con perforación, con absceso adyacente. 


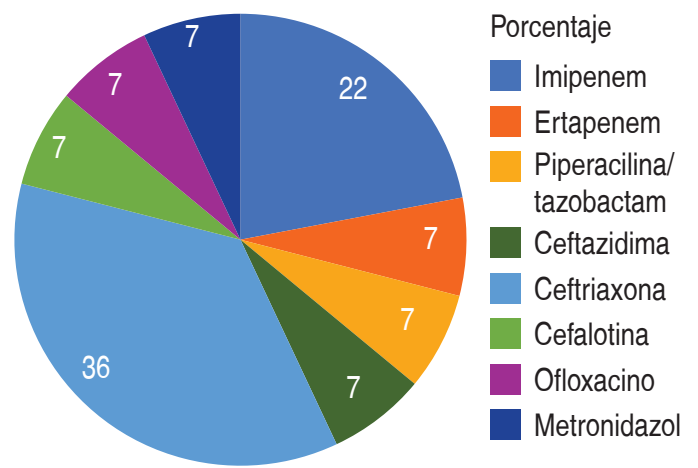

Figura 2: Antibióticos empleados en pacientes quirúrgicos con lesión renal aguda.

modo de monoterapia iniciada como esquema prehospitalario. En la etapa de hospitalización, se cambió a un fármaco de mayor dispersión siguiendo las recomendaciones de guías internacionales; en esta serie el antibiótico iniciado fue imipenem (21.4\%) (Figura 2). En días, la duración media de terapia antimicrobiana fue de 23.4, sin embargo la media del lapso de estancia intrahospitalaria fue de 18.2, lo cual representa un periodo largo de tiempo. Finalmente, en la resolución quirúrgica, el $60 \%$ precisó drenaje percutáneo y sólo un $40 \%$ fue candidato a laparoscopia.

El reporte del antibiograma demostró más de dos cepas multirresistentes, y el germen aislado con mayor frecuencia fue Escherichia coli BLEE (40\%) seguido de una infección polimicrobiana $(40 \%)$ (Tabla 3).

\section{DISCUSIÓN}

La severidad de la LRA aumenta en la disfunción multiorgánica y se correlaciona con el estado de choque. En los factores pronósticos de mala evolución se encuentra como mayor predictor la edad del enfermo con sus comorbilidades, dado que en estudios multicéntricos y experimentales se ha demostrado la fuerte asociación que hay entre distintas enfermedades que tienen por fisiopatología estados hipoglucémicos, desarreglo endotelial e inmunosupresión, habida cuenta de que aumentan la vulnerabilidad del paciente ante algún proceso infeccioso y eventualmente sepsis. Por otra parte, dentro de los elementos protectores se halla un descenso rápido en las primeras 24 horas de hospitalización. ${ }^{10-14}$

Para valorar la gravedad, existen escalas empleadas dentro del abordaje inicial que han sido estudiadas, validadas con alta sensibilidad, y de fácil y rápida obtención buscando que los afectados puedan beneficiarse precozmente del diagnóstico y por supuesto del tratamiento antibiótico más adecuado. ${ }^{15}$ Entre ellas están SOFA y qSOFA, las cuales han demostrado ser útiles para brindar un pronóstico y son utilizadas frecuentemente en la valoración de los pacientes quirúrgicos.

En la casuística que aquí se presenta, la mayoría de los enfermos ingresados tuvieron criterios de choque séptico severo y por tanto esto explica la alta proporción de afectados, quienes requirieron reanimación hídrica, apoyo farmacológico usando vasopresores e indicación precoz de antibioticoterapia de amplio espectro. ${ }^{16}$ Las más actuales recomendaciones de uso de antibióticos se basan en gravedad leve, moderada y severa. En los dolientes quienes proceden de la comunidad, con signos clínicos de peritonitis, pero que no reúnen pautas de sepsis grave y aún no han recibido terapia antibiótica, debe ofrecerse la asociación de una cefalosporina de tercera generación utilizando metronidazol; si son alérgicos a los $\beta$-lactámicos puede asociarse una quinolona. ${ }^{17}$

En aquellos quienes proceden de la comunidad, sin infección severa pero que ya recibieron antibióticos, debe sospecharse la presencia de enterobacterias BLEE. Si no hay

\section{Tabla 3: Espectro bacteriológico reportado por antibiograma. $\mathrm{N}=5$.}

\begin{tabular}{ccc} 
Paciente & Reporte de cultivo & Antibiograma \\
\hline 1 & Multimicrobiana & $3 \mathrm{MDR}$ \\
2 & E. coli BLEE & $2 \mathrm{MDR}$ \\
3 & S. pyogenes & $4 \mathrm{MDR}$ \\
4 & E. coli BLEE & $3 \mathrm{MDR}$ \\
5 & Multimicrobiana & $1 \mathrm{MDR}$ \\
\hline
\end{tabular}

E. coli BLEE: Escherichia coli productor de betalactamasas de espectro extendido, $S$. pyogenes = Streptococcus pyogenes, MDR = multidrogorresistencia. 
riesgo de contaminación por Pseudomona aeruginosa, puede administrarse ertapenem como monoterapia. Y finalmente, los sujetos con criterios de condición séptica grave serán quienes deban recibir una combinación antibiótica de amplio espectro. ${ }^{18}$

En cuanto al rango microbiológico reportado acerca de las IIA complicadas, en la bibliografía internacional se informa sobre el predominio de bacilos gramnegativos, por ejemplo Escherichia coli (25-30\%), seguido de Klebsiella spp y Pseudomona aeruginosa (3-6\%). En relación con los cocos grampositivos destacan: Streptococcus spp (16\%), Staphylococcus spp (5.2\%) y en menor medida, Enterococcus spp (4.7\%). ${ }^{19,20}$ Es importante ya que, en el estudio realizado, la mayoría de las IAC son multidrogorresistentes y polimicrobianas, lo cual arroja un diagnóstico bacteriológico amplio y difícil de erradicar con un tratamiento con periodos de terapia estandarizados de pocos días, se tiene que hacer todo lo contrario: ampliar y siempre tener en cuenta el hecho de la farmacocinética y dinámica de aquellos antibióticos prescritos, para no causar mayor daño que beneficio al paciente. ${ }^{21}$ Después de la recuperación de la sepsis, los enfermos aún son susceptibles al deterioro de la salud, el $40 \%$ es internado dentro de los siguientes 90 días. ${ }^{19,22}$

La atención hospitalaria precoz de la infección se centra en el reconocimiento rápido, el tratamiento utilizando antibióticos de amplio espectro, la eliminación de las fuentes de contaminación, todas estas estrategias mejoran la calidad de egreso del paciente y en general de la función orgánica comprometida por la infección.

\section{CONCLUSIÓN}

En el contexto de un enfermo con IIA complicada con sepsis grave y además LRA, se vuelven imprescindibles la evaluación de la severidad y la adopción de medidas que conducen a una disminución de la mortandad y los costos asociados con el tratamiento y la hospitalización.

La condición séptica tiene incidencia y mortalidad elevadas, por ello, la antibioticoterapia debe ser elegida de acuerdo a la gravedad y estado clínico, de laboratorio y de la disponibilidad de los fármacos, permitiendo que la decisión médica sea basada en evidencia.

\section{REFERENCIAS}

1. Bulander RE, Dunn DL, Beilman GJ. Schwartz's Principles of Surgery 2. Chapter 6. Surgical Infections. McGraw Hill. 2019, 113-130.

2. Rattan R, Allen CJ, Sawyer RG, Askari R, Banton KL, Claridge JA, et al. Patients with complicated intraabdominal infection presenting with sepsis do not require longer duration of antimicrobial therapy. J Am Coll Surg. 2016; 222: 440-446.

3. Sartelli M, Catena F, Ansaloni L, Coccolini F, Di Saverio S, Griffiths EA. Duration of antimicrobial therapy in treating complicated intra-abdominal infections: a comprehensive review. Surg Infect. 2016; 17: 9-12.

4. Sood MM, Shafer LA, Ho J, Reslerova M, Martinka G, Keenan S, et al. Early reversible acute kidney injury is associated with improved survival in septic shock. J Crit Care. 2014; 29: 711-717.

5. Thijs A, Thijs LG. Pathogenesis of renal failure in sepsis. Kidney Int Suppl. 1998; 66: S34-37.

6. Zamoner W, De Freitas FM, Garms DSS, Gobo OM, Balbi AL, Ponce D. Pharmacokinetics and pharmacodynamics of antibiotics in critically ill acute kidney injury patients. Pharma Res Per. 2016; 4: 1-7.

7. Sartelli M, Catena F, Barnabé R. Complicated intraabdominal infections worldwide: the definitive data of the CIAOW Study. World Journal of Emergency Surgery. 2014. 9:37. 1-10.

8. Sartelli M, Chichom-Mefire A, Catena F. The management of intra-abdominal infections from a global perspective: 2017 WSES guidelines for management of intraabdominal infections. World Journal of Emergency Surgery. 2017; 29: 1-34.

9. Carrillo R, Carrillo JR, Carrillo LD. Estudio epidemiológico de la sepsis en unidades de terapia intensiva mexicanas. Cir Ciruj. 2009; 77: 301-308.

10. Bernato AE, Alexander SL, Linde ZWT, Angus DC. Racial variation in the incidence care and outcomes of sever sepsis. Analysis of population patient and hospital characteristics. Am J Respir Crit Care Med. 2008; 177: 279-284.

11. Venot M, Weis L, Clec'h C, Darmon M, Allaouchiche B, Goldgran-Tolédano D, Garrouste-Orgeas M, Adrie C, Timsit JF, Azoulay E. Acute kidney injury in severe sepsis and septic shock in patients with and without diabetes mellitus: a multicenter study. PLoS One. 2015; 10: e0127411. doi: 10.1371/journal.pone.0127411.

12. Spiller F, Carlos D, Souto FO, de Freitas A, Soares FS, Vieira SM, et al. $\alpha 1$-acid glycoprotein decreases neutrophil migration and increases susceptibility to sepsis in diabetic mice. Diabetes. 2012; 61: 15841591.

13. Korbel L, Spencer JD. Diabetes mellitus and infection: an evaluation of hospital utilization and management costs in the United States. J Diabetes Complications. 2015; 29: 192-195.

14. Machado-Villarroel L, Montano-Candia M, DimakisRamirez DA. Diabetes mellitus y su impacto en la etiopatogenia de la sepsis. Acta Méd Grupo Ángeles. 2017; 15: 207-215.

15. Godínez-Vidal AR, García-Vivanco DM, MonteroGarcía PJ, Martínez-Martínez AR, Gutiérrez-Banda CA, Gracida-Mancilla NI. Utilidad del índice SOFA 
en sepsis abdominal por peritonitis secundaria. Rev Hosp Jua Mex. 2018; 85: 195-200.

16. Sartelli M, Catena F, di Saverio S, Ansaloni L, Coccolini F, Tranà C, Kirkby-Bott J. The challenge of antimicrobial resistance in managing intra-abdominal infections. Surg Infect. 2015; 16: 213-220.

17. Guirao X, Arias J, Badía JM, García-Rodríguez JA, Mensa JJ, Álvarez-Lerma JJ, et al. Recomendaciones en el tratamiento antibiótico empírico de la infección intraabdominal. Cir Esp. 2010; 87: 63-81.

18. Montravers P, Lepape A, Dubreuil L, Gauzit R, Pean Y, Benchimol D, Dupont H. Clinical and microbiolocal profiles and community-acquired and nosocomial intra-abdominal infections: results of the French prospective, observational EBIIA study. J Antimicrob Chemother. 2009; 63: 785-794.

19. Liñán-Ponce JI, Véliz-Vilcapoma F. Clinical characteristics of the admitted patients with severe sepsis to an Intensive Care Unit. Rev Soc Peru Med. 2008; 21: 121-127.

20. Bisso AA. Antibioticoterapia en las infecciones graves. Acta Méd Peruana. 2011; 28: 27-38.

21. Meregalli A, Oliveira RP, Friedman G. Occult hypoperfusion is associated with increased mortality in hemodynamically stable, high-risk surgical patients. Crit Care. 2004; 8: R60-R65.
22. Regueira T, Andresen M, Mercado M, Downey P. Fisiopatología de la insuficiencia renal aguda durante la sepsis. Med Intensiva. 2011; 35: 424-432.

Consideraciones éticas: Los procedimientos en humanos deben ajustarse a los principios establecidos en la Declaración de Helsinski de la Asociación Médica Mundial (AMM) y con lo establecido en la Ley General de Salud Título Quinto y Reglamento de la Ley General de Salud en Materia de Investigación para la Salud, y NOM012-SSA3-2012, que establecen los criterios para la ejecución de proyectos de investigación para la salud en seres humanos, así como con las normas del Comité de Ética en Investigación de la institución donde se efectúen.

Correspondencia:

Yeiscimin Sánchez Escobedo

Adolfo López Mateos Núm. 40,

San Pedro, Puebla, Puebla,

Tel: 7971218088

E-mail: yeiscimin@gmail.com 